\title{
Comparing Successful DLT Consortia: A Lifecycle Perspective
}

\author{
Benedikt Putz \\ University of Regensburg \\ benedikt.putz@ur.de
}

\author{
Günther Pernul \\ University of Regensburg \\ guenther.pernul@ur.de
}

\begin{abstract}
In 2021, enterprise distributed ledger technology has evolved beyond the proof-of-concept stage. It is now providing business value to large consortia in several successful and well documented case studies. Nevertheless, other consortia and initiatives are stuck in early stages of consortium formation or conceptualization. They stand to benefit from lessons learned by successful consortia, but an in-depth comparison has not yet been conducted. Thus, this study performs the first methodological comparison of large DLT consortia that have launched a product. Based on the temporal evolution of these consortia, a lifecycle with 4 stages and 12 sub-phases is developed to provide further guidance for early-stage consortia. The results show how 9 pioneer consortia have successfully integrated novel DLT into existing processes, but also point out challenges faced on the way.
\end{abstract}

\section{Introduction}

Distributed Ledger Technology (DLT) offers great potential to improve inter-organizational processes by improving information transparency, traceability, efficiency and ultimately reducing costs $[1,2]$. Despite the promised benefits, it has not yet reached widespread adoption. DLT faces numerous technical challenges with regard to scalability, privacy and interoperability among others [3]. But these are not the main issue preventing adoption. Indeed, the main challenge for businesses looking to reap DLT's benefits is successful collaboration with ecosystem partners $[4,5]$. A global survey with 1400 respondents from businesses around the world in March 2020 further confirms this assessment [6]. $30 \%$ of its respondents agree to face challenges in forming a consortium for DLT-based collaboration. In addition, $40 \%$ face at least one of several challenges participating in the consortium collaboration (such as defining balanced governance rules, defining roles and responsibilities, cross purposes of members). Consequentially, despite significant progress in some industries, many initiatives remain stuck in a proof of concept stage and are thus investing without gaining business benefits. Still, there are several pioneers that have used the technology successfully to improve business processes. To date, researchers have only published isolated studies of such cases $[7,8,9]$. Going beyond single case studies, multiple case study designs can provide more robust results by revealing findings through replication logic [10]. These findings may prove useful to newer DLT consortia in early stages of development. To our knowledge, no such study has been conducted with regard to DLT consortia. Therefore, we derive the following research questions:

RQ1: What are the commonalities and differences between operational DLT consortia?

RQ2: Which phases does a DLT consortium undergo during its lifecycle?

Contribution. To answer these questions, we conduct a systematic multiple case study of successful production use cases of DLT in a consortium setting. To our knowledge, this is the first systematic study of DLT consortia that have launched an operational network. Besides determining commonalities and differences, we also focus on the temporal dimension of consortium building by developing a lifecycle from empirical evidence. We contribute to theory by aggregating and comparing evidence from distinct DLT case studies, while building new theory for future empirical studies with the lifecycle. Our study contributes to practice by providing a guideline for DLT consortia in early adoption stages, which allows them to tackle governance issues in a structured way.

This paper is organized as follows: Section 2 provides an overview of related research with regard to consortium collaborations and blockchain governance. Section 3 explains our methodology for case study selection and lifecycle development. It also provides reasoning for the selection criteria mentioned in the previous paragraph. In Section 4, the selected cases are briefly introduced. Based on an in-depth review 
of each case, Section 5 focuses on answering RQ1, while Section 6 introduces the DLT consortium lifecycle answering RQ2. Finally, we discuss our findings and limitations in Section 7 and wrap up with a summary of the results in Section 8.

\section{Background}

While literature commonly uses the term blockchain, this paper uses the term DLT, which covers a broader range of technical frameworks (i.e. R3 Corda is technically not a blockchain [11]). We also use the term consortium for a DLT-based partner network, defined as "a form of cooperation between institutions that see value in sharing resources and know-how to save costs" [7]. In particular, business-oriented consortia focus on solving business problems with DLT, as opposed to technology-oriented consortia, which focus on developing DLT platforms (i.e. R3, Hyperledger). This study focuses on business-oriented consortia.

Before explaining our methodology, we give some background on information sharing in partner networks, which is currently the main business objective of DLT consortia. Regarding blockchain and DLT, the research stream blockchain governance focuses on the processes and decision rights in DLT consortia and is thus closely related to the present work.

\subsection{Information sharing in partner networks}

A network of collaborating business partners is the foundation necessary to begin information sharing with a technological artifact. Such partner networks are typically formed in stages, which are identified by Larson et al. and shown in Figure 1 [12]. The process starts with the trial period, where companies exchange initial information about each other's knowledge, capabilities and resources. First experiences are made based on initial collaborations, thus generating trust. Once enough trust has been built up, companies proceed to an integration period, where companies begin having increasing influence over others in their interactions. More risks are taken and short-term losses are more easily accepted with the expectation of long-term profits. In this period, processes are more closely aligned to reduce organizational differences [13]. This also includes closer integration of technical infrastructure, which improves the effectiveness of the collaboration by increasing transparency. Larson refers to this constant improvement as "Kaizen".

DLT is a technological artifact that can be used to significantly enhance the technical integration. DLT removes the need to trust one partner or outsider with data management, while providing non-repudiation,

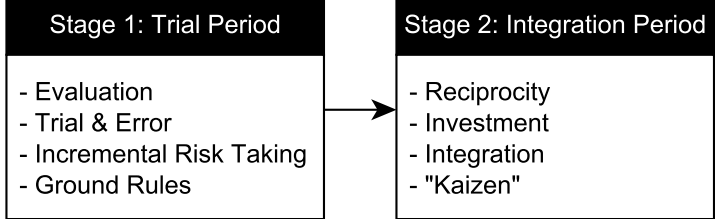

Figure 1: Stages of the business network formation process [12]

integrity and transparency guarantees [14].

Once partners are sufficiently integrated, the network is established (network phase). A functioning network is characterized by an atmosphere of trust, which enables open communication and collaborative problem solving. While DLT cannot guarantee this trust, it can enable it by providing a stable technological basis that even permits isolated misbehavior under an honest majority [15].

\subsection{Blockchain Governance}

First and foremost, blockchain governance research deals with the distribution of responsibilities and power among consortium participants. This includes decision-making rights and processes and accountability of decision-makers [16].

One stream of research focuses on developing layers and dimensions for governance of blockchains [17, 18]. Pelt et al. focus mostly on permissionless blockchains (public networks without access restrictions) and their open-source governance. They describe three layers: Off-chain community, off-chain development and on-chain protocol. Dimensions further describe the aspects of each layer [17]. The approach by Beck et al. instead focuses on permissioned networks (closed networks focused on enterprise usage), establishing incentives, accountability and decision rights as core dimensions for blockchain governance [18].

Ziolkowski et al. examine decision problems in blockchain governance, providing guidance on the handling of challenging decisions faced by the interview participants $[16,2]$. Their case study interviews were conducted in 2017, so most of the cases were still creating proof-of-concept prototypes. In addition, there are many case studies focusing on the development and challenges faced by a single consortium, i.e. TradeLens [8], Cardossier [7] and MediLedger [9]. The University of Cambridge conducted its latest benchmarking study on live DLT networks in 2019 [19]. However, the study is missing a transparent and methodological approach to consortium selection. The lack of consideration 
for consortium size leads to skewed results in two ways. Firstly, the study itself categorizes $77 \%$ of its database as "blockchain memes", i.e. systems without real multi-party consensus. Secondly, results are biased toward individual companies developing solutions $(71 \%)$ instead of consortia.

Few existing studies consider the temporal dimension of consortium development. Especially regarding permissioned blockchains, there is still uncertainty regarding the formation and development processes of blockchain consortia. The Cambridge Benchmarking Study proposes four stages (exploration, concept, trial, production), but does not detail how these stages were determined or relate them back to individual cases [19]. The study also does not investigate in detail what happens in each phase and what happens beyond the initial production deployment. Deventer et al. created a strategic options model with exploration and exploitation phases, but this was only based on a conceptual experiment [20]. Therefore, this study fills the gap by methodologically deriving a holistic lifecycle of blockchain consortium development from empirical evidence, from consortium formation to post-launch expansion.

\section{Methodology}

Based on the initial model derived from business network formation (see Figure 1), we further refine this model based on a comparative multiple case study research design [10]. We perform a holistic study of multiple cases, where each case represents a different context in which DLT is being adopted. The study aims to answer our research questions RQ1 and RQ2, motivated by the lack of cross-case comparisons for DLT consortia in the literature.

We first conduct a literature review of existing DLT use cases to determine applicable cases for our study. To find as many cases as possible we conducted a breadth-first search, which included:

- DLT and industry news websites (LedgerInsights ${ }^{1}$, Global Trade Review ${ }^{2}$, Forbes Blockchain 50 [21]

- DLT framework websites (i.e. Hyperledger, R3, Multichain use case sections)

- industry review studies (WTO study on DLT in Global Trade [22])

Overall, the breadth-first search yielded a total of 35 DLT consortia in various stages of development. To deepen our view of each case, we retrieve available

\footnotetext{
$1_{\text {wWw.ledgerinsights.com }}$

$2_{\text {www.gt review.com }}$
}

information from the web. We search for mentions in scholarly literature using the databases Google Scholar, IEEE Xplore and Scopus and combining the consortium brand name (column 1 in Table 1) with the term "case study". Other valuable sources include the consortium website, consortium partners' websites, press interviews and news articles.

A critical part of this work is defining a successful case. We consider a case successful iff

1. it has been operating live for at least 6 months

2. at least 7 participants operate DLT nodes

The period of live operation is determined based on the point of live deployment in the unified software development process [23]. We use 6 months as the lower bound to be able to investigate post-launch phases. 7 nodes is the lower bound to tolerate at least 2 dishonest nodes in the $3 f+1$ byzantine fault-tolerant consensus model [15]. As trust issues among participants are a key point on the decision path to DLT adoption [14], tolerating some misbehavior is essential to build a successful platform. In combination with 6 months of live operation, the lower bound on network nodes also serves to ensure that some business value is being delivered, as otherwise organizations would begin leaving to save costs.

Additionally, to ensure sufficient empirical evidence for analysis we define a structured set of criteria based on the well-known Information Quality (IQ) Framework [24]. We focus on Intrinsic IQ and Contextual IQ, where we consider the following characteristics:

Intrinsic IQ: accuracy, objectivity, believability, and reputation of data

Contextual IQ: appropriateness of contextual parameters of data for the task at hand

Therefore, Intrinsic IQ is based on the quality of associated publications. We consider a case's intrinsic IQ as high if there is at least one publication with Q1/Q2 ranking in ISI WoK Journal Rankings ${ }^{3}$, or at least B quality in CORE conference/journal rankings ${ }^{4}$. We consider it medium, if there are at least 5 different sources of acceptable quality (white papers, online case studies, news articles, website entries).

Contextual IQ is used to measure the completeness of the case study based on publicly available data, as well as its appropriateness based on the consortium's success. This metric is computed as the percentage of

\footnotetext{
${ }^{3}$ http: / / www.webofknowledge.com/JCR

${ }^{4}$ https: / / www.core.edu.au/conference-portal
} 
Table 1: Overview of selected case studies (in alphabetical order).

\begin{tabular}{lllllrll} 
Name & Lead & Partner & Platform & Sector & Nodes & Legal Form & Country \\
\hline B3i Re & B3i Services & R3 & Corda & Insurance & 21 & Stock Corp. & Switzerland \\
\hline Bakong & NBC & Soramitsu & Iroha & CBDC & 16 & led by NBC & Cambodia \\
\hline Cardossier & cardossier & AdNovum & Corda & Vehicles & 8 & Association & Switzerland \\
\hline Contour & Contour & Contour & Corda & Trade Finance & 8 & Limited & Singapore \\
\hline covantis & Covantis & ConsenSys & Quorum & Agriculture & 18 & SA & Switzerland \\
\hline DL Freight & Walmart Canada & DLT Labs & Fabric & Logistics & 30 & Incorporated & Canada \\
\hline MediLedger & Chronicled & Chronicled & Ethereum & Pharma & 10 & Incorporated & US \\
\hline TradeLens & Maersk & IBM & Fabric & Logistics & 14 & Subdivision & US \\
\hline we.trade & we.trade & IBM & Fabric & Trade Finance & 16 & DAC & Ireland \\
\hline
\end{tabular}

pertinent questions that we were able to answer based on available sources. Specifically, we consider the following questions:

Q1: Is sufficient information consortium evolution available (trial/integration phases)?

Q2: Did the consortium platform launch at least 6 months ago?

Q3: Do at least 7 participating organizations control DLT nodes?

Q4: Could the name of the used DLT platform be extracted?

Cases were included in our study if they have an intrinsic IQ of at least medium and a contextual IQ of $100 \%$. After applying this filter, 9 cases remain, which we focus on in the following sections. The full table of gathered data on consortia is available online, along with intrinsic and contextual IQ filter results ${ }^{5}$.

For lifecycle development, we follow a bottom-up, empirical and iterative process. Figure 1 from Section 2.1 provides initial guidance on the trial and integration phases. We subsequently refine these phases based on common milestones of each consortium (see Table 2) and activities occurring between these milestones (see Table 3). Finally, we performed several semi-structured expert interviews with consortium representatives to validate our findings.

\section{Selected Cases}

An overview of selected case studies is given in Table 1. Hereafter, a brief description of each selected

\footnotetext{
5https://drive.google.com/drive/folders/ 1M3046mb6X1UsxnR91_dj80 jXzJXRiivb
}

case study is given, followed by a citation of the primary source(s) for the case.

B3i Reinsurance. B3i refers to the Blockchain Insurance Industry Initiative, a consortium jointly currently owned by 21 insurance companies. The consortium develops solutions for the insurance market, with its current solution B3i Reinsurance (B3i Re) focused on reinsurance Catastrophe Excess of Loss (Cat $\mathrm{XoL}$ ) treaties. Primary source for B3i Reinsurance is the case study by its implementation partner R3 [25], accompanied by a large number of detailed blog posts on their website.

Bakong. Bakong is an initiative of the National Bank of Cambodia (NBC), and one of the first Central Bank Digital Currencies (CBDC) to launch in production on Hyperledger Iroha in June 2020. Its stated objective is improving financial inclusion across Cambodia through simplified access to bank accounts and near real-time mobile payments. Primary source is the Bakong Guide Book published by the NBC [26].

Cardossier. The Cardossier consortium was built with the goal of establishing a single source of truth for car-related data across a car's lifecycle. It currently has 20 members, including government organizations and businesses from the automotive, finance and insurance sectors. It runs in production on the Corda DLT since June 2020. The primary source is the case study by Zavolokina et al. [7]. In addition, this consortium is noteworthy for its double-digit count of published research papers, thanks to its collaboration with the University of Zurich.

Contour. Contour is a Trade Finance platform focused on digitizing Letters of Credit. It went live on an R3 Corda network in October 2020. R3 also provides a case study focused on Contour, which is our primary source [27]. 
Covantis. Covantis aims to digitize processes in agricultural trading and shipping by creating a trusted single source of information. It relies on the ConsenSys-supported Quorum blockchain platform. ConsenSys has published a case study that serves as our primary source [28].

DL Freight. DL Freight was built by DLT Labs for Walmart Canada to address freight invoicing issues for logistics carriers. The platform is based on Hyperledger Fabric and runs in production since October 2019. Primary source is a case study conducted by the University of Arkansas [29].

MediLedger. MediLedger is a US-based consortium focusing on product traceability and preventing counterfeits in the pharmaceuticals supply chain. It was founded in 2017, and went into production on Parity Ethereum in October 2019. Primary source is the case study by the University of Bamberg [9].

TradeLens. TradeLens is a supply chain platform focused on digitizing bills of lading for container shipping. It was founded by Maersk, starting with research prototypes of different names as early as 2013. The decision to commercialize and launch it as the TradeLens platform was made in December 2018. Since then, it has grown to become the largest DLT platform by ecosystem size, currently claiming more than 200 members globally. Primary source for TradeLens is the case study conducted by Jensen et al. [8].

we.trade. we.trade is a platform focused on trade finance, specifically on Bank Payment Undertakings (buyers providing bank payment guarantees to sellers). It was founded at the beginning of 2017 as a joint venture between 12 European banks and subsequently went into production in October 2018. Primary source is the case study by its technology provider IBM [30], complemented by interviews on Global Trade Review for more recent developments since 2018.

For brevity, we refer to the cases described in this chapter simply as cases or consortia in the following sections.

\section{Case Comparison}

In this Section, we attempt to answer our first research question based on the findings from the case studies: RQ1: What are the commonalities and differences between operational DLT consortia?

Specifically, we analyze six dimensions derived from blockchain governance literature and our own research: Platform choice [19], Network size [15], Incentives [17, 18], Legal Form [18], Disintermediation [31, 4] and Interoperability [19, 4].

Platform choice. The choice of DLT platform
Table 2: Milestones of the selected consortia.

\begin{tabular}{lrrrr} 
Name & Foundation & \multicolumn{1}{l}{ Instit. } & \multicolumn{1}{l}{ Pilot } & Launch \\
\hline B3i Re & Oct-16 & Mar-18 & Jun-18 & Jul-19 \\
\hline Bakong & - & - & Jul-19 & Oct-20 \\
\hline Cardossier & Nov-16 & Mar-19 & Apr-19 & Jun-20 \\
\hline Contour & Jul-17 & Jan-20 & May-19 & Oct-20 \\
\hline covantis & Oct-18 & Mar-20 & - & Feb-21 \\
\hline DL Freight & - & - & Jan-19 & Oct-19 \\
\hline MediLedger & Jan-17 & - & Feb-19 & Oct-19 \\
\hline TradeLens & Jan-17 & Aug-18 & Jan-17 & Dec-18 \\
\hline we.trade & Jan-17 & Apr-18 & Jun-18 & Oct 18 \\
\hline
\end{tabular}

differs among consortia. Hyperledger Fabric is the most popular framework (4 consortia), closely followed by Corda (3 consortia). Ethereum and Hyperledger Iroha support 1 consortium each. Regarding the full list of 33 consortia, the distribution changes. 16 consortia use Hyperledger Fabric and only 6 Corda. Quorum is used by 5, with others (i.e. Multichain, Ethereum, Iroha) totaling 6 . Corda is almost exclusively used by Trade Finance and Insurance consortia, with Cardossier marking the exception. Overall, Hyperledger Fabric seems to be the most flexible platform fitting most use cases, but other platforms have valid use cases as well.

Network size. The network size refers to the number of distinct independent organizations operating the underlying DLT network (i.e. taking on a validator role). The size of the selected consortia varies between 8 (Contour) and 30 (DL Freight). Member counts published in official press releases must be carefully considered. For example, TradeLens claims more than 200 ecosystem members, but only a small number of those are actually performing validator roles on the DLT. For TradeLens, validators are referred to as "trust anchors", a role currently assumed by ocean carriers. 14 ocean carriers and an additional node operated by consortium's legal entity perform consensus validation for the network. Other ecosystem partners have much less control and only interact with the DLT via an API.

Incentives. Initially, incentives refer to reasons for new members to join the network. For marketing these incentives, the consortia focus on the actual business benefits that the solution provides - usually digitized and more efficient processes, which result in cost savings. The fact that DLT is used for this purpose is not specially emphasized, unless information technology executives are addressed directly. Some consortia still mention it at the same level with other business benefits (Cardossier, MediLedger). However, as other researchers have noted 
[4], incentives work only if they represent business value for potential members ("executives don't care about blockchain").

Legal Form. There is no clear single best choice for the legal form of a DLT consortium. Most cases choose for-profit entities, such as Corporation, Limited or as a subdivision of an existing legal entity. For example, it was decided to make the TradeLens operating company a fully owned subsidiary of Maersk to ease legal approvals in countries around the world [8]. However, the more frequent case is incorporation of a new company where consortium members become shareholders (we.trade, B3i, Contour). Cardossier marks the exception by founding a non-profit association tasked with maintaining the consortium platform.

Disintermediation. One of the most frequently cited benefits of DLT is disintermediation. However, recent research has questioned the degree to which DLT actually accomplishes the task of eliminating the platform operator, calling it the "disintermediation fallacy" [31]. Our results partially support this hypothesis. Every reviewed case relies on an external company for A) platform development, B) platform hosting or C) both of these. While for case B the involvement of the third party is limited to infrastructure, in the other case more significant trust is required in the platform developer (and operator in case C). In these cases it is worth questioning if DLT is actually needed to support the platform, since the external platform operator already has sufficient trust and control to invalidate the disintermediation argument.

Interoperability. In some industries, there are several competing DLT consortia which serve similar business needs. This is especially apparent for Global Trade, where multiple operational and emerging platforms serve similar needs (i.e. Bill of Lading, Letter of Credit). In turn, interoperability is becoming a more important concern for platform operators. For example, TradeLens and we.trade built a bridge for trusted data transfer between the two networks as part of a research project [32]. While interoperability is not a primary concern before launch, it should be considered in strategic plans [8]. Especially if there are other DLT networks fulfilling similar needs, interoperability pilots should be conducted. These may even result in mergers which benefit both participants, as was the case when we.trade and Batavia merged in October 2018. DLT platforms are about network effects [33], so greater reach benefits all participants.

By analyzing six dimensions we have now answered RQ1. The results established some commonalities: successful consortia focus on business benefits as incentive for participation, they rely on trusted providers for DLT operation, and favor interoperability initiatives in long-term plans. As for differences, platform choice, network size and legal form are quite heterogeneous. They mostly seem to depend on the specific requirements of the case study and its industry.

\section{DLT Consortium Lifecycle}

This Section is dedicated to answering $\boldsymbol{R Q 2}$ : Is there a common lifecycle for DLT consortia?

As mentioned in Section 3, we iteratively develop a lifecycle based on a detailed review of all selected cases. Table 2 shows the four milestones Founding, Institutionalization, Pilot and Launch for each consortium:

- Founding: first consortium announcement

- Institutionalization: legal entity creation

- Pilot: last pilot experiment

- Launch: launch announcement

Based on these milestones, we structure the lifecycle along the Trial and Integration phases detailed in Section 2. The result is shown in Figure 2. Initially, the Formation and Pilot phase involve incremental risk taking and trial \& error during the evaluation of different concepts and pilots. Once sufficient trust is established among consortium partners, consortia proceed to the Launch and Expansion phases. Each of these phases consists of multiple sub-phases, which are not necessarily sequential in order. The lifecycle is meant to depict the most common approach. If there is an exception to this approach, it is mentioned in the description of the phases hereafter.

\subsection{Formation}

Any DLT consortium is at some point initiated by a single organization reaching out to others for collaboration. This organization is hereafter referred to as consortium initiator. The initiator often becomes the leading driver (leader) of the consortium (Bakong, TradeLens, DL Freight). In other cases, the leader is a newly formed entity determined by consortium shareholders (Cardossier, covantis, we.trade).

Prototyping. The consortium initiator initially develops a concept and vision for the platform. This vision is subject to change during later collaboration with other participants, but it encompasses the basic foundation of the collaboration. This idea is often related to exchanging business documents digitally based on DLT, to improve auditability and traceability. This phase may include proof of concepts 


\begin{tabular}{|c|c|c|c|}
\hline Formation & Pilot & Launch & Expansion \\
\hline \multicolumn{4}{|c|}{ Consortium Building and Governance } \\
\hline & \multicolumn{3}{|c|}{ Iterative Software Development } \\
\hline
\end{tabular}

Figure 2: DLT consortium lifecycle phases.

Table 3: Phases and sub-phases of the consortium lifecycle.

\begin{tabular}{l|l|l} 
Phase & Sub-phases & Description \\
\hline Formation & $\begin{array}{l}\text { Prototyping } \\
\text { Partner Search } \\
\text { Institutionalization }\end{array}$ & $\begin{array}{l}\text { Ideation and first software prototype concepts } \\
\text { Initiator searches for consortium and implementation partners } \\
\text { A legal entity is formed to represent the consortium (not applicable for } \\
\text { pre-existing partner networks) }\end{array}$ \\
\hline Pilot & Requirements Engineering & $\begin{array}{l}\text { Requirements and an initial scope for the business case are established. } \\
\text { This includes compliance and security-based requirements } \\
\text { Prototype software is developed by the consortium institution and/or } \\
\text { software provider } \\
\text { Prototype is used in an actual business process for testing purposes }\end{array}$ \\
\hline Launch & $\begin{array}{l}\text { Production Use } \\
\text { Commercialization }\end{array}$ & $\begin{array}{l}\text { First product is launched for production use } \\
\text { Revenue model developed and consortium institution begins earning } \\
\text { revenue (only for-profit consortia) } \\
\text { Participants are seeing first benefits as a result of adoption }\end{array}$ \\
\hline Expansion & Ecosystem Building & $\begin{array}{l}\text { More peripheral organizations in the supply chain join the platform } \\
\text { (often as users, not operators) } \\
\text { Additional business cases (products) within the consortium are } \\
\text { developed and launched }\end{array}$ \\
\hline
\end{tabular}

to demonstrate the feasibility and potential benefits of a collaboration among the initial partners.

Partner Search. The consortium lead focuses on acquiring suitable partners for the use case. Convincing partners can be the most difficult part of building a consortium, especially if these partners are competitors. An integral part of the trial period is building trust in partners, especially in the consortium lead and that it will be able to navigate ecosystem tensions [8]. During this phase, it can be helpful to focus on first acquiring partners that are not direct competitors, which builds trust initially [7]. While the partner search activity begins in the formation phase, it continues throughout all later phases as the consortium continually seeks to grow its reach. This is fact is represented in Figure 2 as Consortium Building.
Institutionalization. Eventually, newly formed consortia are confronted with the need for a legal entity. The Cardossier project identified several reasons related to obtaining a critical mass and network effects, as well as complying with laws and regulation [7]. As mentioned previously in Section 5, the specific legal form varies. The branding often changes along with institutionalization. For example, we.trade rebranded from its original name Digital Trade Consortium, Contour from Voltron, and TradeLens received its current name on commercialization after multiple renamings [8]. Previously existing business networks (Bakong) or networks initiated and led by a software service provider (MediLedger, DL Freight) do not face this need, as the consortium initiator takes on the role of consortium representation. While Institutionalization is 
part of the Formation phase, it often takes place during the Pilot phase or even during the Launch phase (before the launch date). This can be attributed to the fact that establishing a joint entity as shareholders requires some trust building.

\subsection{Pilot}

The Pilot phase begins with development of the pilot software and finishes with the end of the last process experiment. A pilot launch refers to a limited roll-out of the target platform. In most cases the prototype used for this purpose is feature-complete, and some projects refer to it as the Minimum Viable Product (MVP). During the Process Experiment it is made available to a small set of test partners. Depending on the success and scope of the pilot, multiple pilots may be needed before the project consortium transitions to Launch.

Requirements engineering. The business case is finalized based on the results of Prototyping during the Formation phase. Functional and non-functional requirements of the target DLT platform are determined. To this end, consortium partners collaborate with a software service provider. Requirements are usually based on business and regulatory concerns. Requirements that are common to several reviewed DLT consortia include scalability, privacy and interoperability. MediLedger collaborated with a regulator (the US Food \& Drug Administration) to ensure compliance of the pilot with regulation [9].

Development and Testing. For almost all cases, a single company is tasked with software development in this phase of the project. In some consortia, the newly formed legal entity hires specialized employees itself (Contour). In other cases, the consortium initiator is a software service provider and thus also develops the platform (Cardossier, DL Freight, MediLedger). Finally, others hire service providers specialized for DLT development (TradeLens, we.trade, covantis). Commonly, such service providers also provide a cloud-based platform where node operators can control DLT nodes. This avoids the need to train people in each participating organization on how to set up a DLT node. Another important decision that occurs during this phase is the choice of DLT platform. The initial choice is not final, as pilot results may lead to the decision to switch to another framework better suited to the business case. This phase also marks the beginning of the Iterative Software Development process [23] marked in Figure 2 that continues throughout later phases.

Process experiment. Finally, an experiment is conducted within the target operational business process. This experiment is of limited scope, for example by concerning a specific product in supply chain cases. For one pilot experiment, TradeLens conducted its pilot experiment with the roses supply chain and involved only necessary partners [8]. Trade Finance consortia start with one or multiple pilot financing transactions between banks and businesses. As one example, Contour performed a total of 10 pilot experiments with different partners over the course of 1 year [27].

\subsection{Launch}

When the consortium is satisfied with pilot results, it usually proceeds to launch the full platform for all consortium participants. While the launch itself is tied to a single point in time (see Table 2), it is accompanied by many preparations and post-launch effects, which are addressed in this phase.

Production Use. In practice, members rarely use the platform for all business transactions immediately after the launch date. In most cases, members finish platform onboarding months after the launch announcement. Often there are separate announcements when a member has completed onboarding [8] or their first successful transaction [27]. These delays can be explained by varying commitment levels of the partners, but most importantly by challenges in integrating novel DLT with existing business processes and legacy systems.

Commercialization. First revenue streams are beginning to materialize for the consortium's legal entity. During the first months after launch, platform usage is usually low, as members begin to move more and more transactions to the new system [25]. Revenue models are usually based on recurring membership fees for consortium members [29, 7, 9]. Notably, TradeLens offers free access to some ecosystem members like container terminals and authorities, while others must pay a subscription and transaction fees [8].

Business Value. Consortium members notice first positive returns on their investment. These are use case dependent, but principally include lower delays (trade finance cases), increased supply chain transparency (product tracking cases) and improved process efficiency through digitization of paper-based documents. For example, DL Freight reduced invoice disputes, which lowered accounts receivable for carriers and costs for Walmart [29]. Others like Bakong and B3i cite significant cost and time savings [26, 25].

\subsection{Expansion}

During the Expansion phase, the consortium focuses on increasing business value. This phase begins about 6 - 12 months after the platform has been successfully 
adopted, deployed and used at all members.

Governance. During expansion of the network, new decisions require consensus building among consortium members. These concern

- new member admission

- new feature prioritization

- accommodating regulatory concerns

- platform monetization

- software updates

- handling security incidents

While some rules are part of the initial consortium agreements, these are ongoing concerns that may require intervention during operation. For example, DL Freight requires chaincode software updates to be approved by a majority of participants [29]. In addition, security concerns exist and may be hard to deal with in a cooperative environment [15].

Ecosystem Building. To ease member acquisition, consortia focus on building an ecosystem around the core DLT platform after launch. While most platforms include the most important core features initially, additional integrations with existing systems ease member acquisition. Another focus during this phase is onboarding related actors that need access to trustworthy DLT data, but don't necessarily validate transactions on the platform. These include auditors, government authorities and suppliers/customers of consortium members.

Expansion of Scope. Gradually, consortia agree on adding new features and products to the platform to expand its scope and concomitant business benefits. In addition to incremental improvements, consortia often bundle major features or new products in a major version release. For example, DL Freight and B3i have announced 2.0 versions of their platforms with significantly expanded capabilities.

\section{Discussion}

Democratic governance vs. benevolent dictators. Democratic decisions are important for consortium longevity and trust-building. For example, for TradeLens the decision-making changed from the platform owner making all the decisions to a more democratic model governed by an advisory board [8]. While some consortia favor democratic decisions, others prefer a "benevolent dictator" approach [9]. In DL Freight, while chaincode updates require approval by carriers, the core direction of the platform is determined by Walmart Canada and DL Freight [29]. Cardossier followed an hierarchical off-chain governance model initially, but plans to transition to a more democratic model in the long-term [7]. To summarize, initially a more hierarchical approach can help consortia move quickly toward Pilot and Launch, but during the Expansion phase democratic governance is preferable to ensure stakeholder expectations are met.

Disintermediation. Both theory and practice have long claimed that DLT disintermediates trusted third parties (TTPs) [4, 31]. However, all consortia in our study agree on the fact that an independent entity is needed to coordinate the consortium's technology development. While this may seem counterintuitive at first, it is a logical consequence of coopetition in partner networks. Practitioners have noted that the need for competitors to cooperate is one of the hardest challenges to solve, often requiring a sponsor such as an industrial body to step in [5]. For some consortia like Bakong, there is a natural sponsor (NBC). For others, new institutions are created for this purpose as TTPs, which are controlled by the members as shareholders. Whether the TTP's software platform must be DLT-based remains controversial, as consortia like Komgo ${ }^{6}$ have transitioned away from DLT entirely in favor of a centralized database. As the blockchain/DLT buzzword slowly loses its appeal (cf. Incentives dimension in Section 5), the focus is now on realizing actual business benefits of DLT.

Study limitations. Three consortia were not included since they failed to meet Q2, so they may be included in future studies. Several other candidates were not included due to Q1, a lack of information available on the development phases. Additionally, there is a slight bias towards western use cases, as many Asian consortia (especially in China) are inaccessible to the authors due to language barriers.

\section{Conclusion}

We performed a multiple case comparative study to find commonalities and differences among successful DLT consortia. Following a strict selection methodology based on contextual and intellectual information quality, 9 successful consortia were selected. The findings were structured along 6 dimensions and used to develop a new lifecycle theory for DLT consortia. They challenge the disintermediation aspect of permissioned DLTs and open up an avenue for future research. They also provide insights for early stage DLT consortia, as well as informing future DLT case studies and DLT platform theory.

Acknowledgment. We would like to thank our interview partners for their valued time and input toward improving data accuracy and the lifecycle.

\footnotetext{
$6_{\text {www.komgo.io }}$
} 


\section{References}

[1] S. Tönnissen and F. Teuteberg, "Analysing the impact of blockchain-technology for operations and supply chain management: An explanatory model drawn from multiple case studies," International Journal of Information Management, 2020.

[2] R. Ziolkowski, G. Miscione, and G. Schwabe, "Decision Problems in Blockchain Governance: Old Wine in New Bottles or Walking in Someone Else's Shoes?," Journal of Management Information Systems, 2020.

[3] S. Meiklejohn, "Top Ten Obstacles along Distributed Ledgers Path to Adoption," IEEE Security Privacy, vol. 16, pp. 13-19, July 2018.

[4] M. Lacity and R. Van Hoek, "What we've learned so far about blockchain for business," MIT Sloan Management Review, vol. 62, no. 3, pp. 48-54, 2021.

[5] B. Carson, G. Romanelli, P. Walsh, and A. Zhumaev, "Blockchain beyond the hype: What is the strategic business value," McKinsey \& Company, pp. 1-13, 2018.

[6] M. Budman, R. Bhat, and S. Bordoloi, "Deloitte's 2020 Global Blockchain Survey - From promise to reality," 2020.

[7] L. Zavolokina, R. Ziolkowski, I. Bauer, and G. Schwabe, "Management, governance, and value creation in a blockchain consortium," MIS Quarterly Executive, 2020.

[8] T. Jensen, J. Hedman, and S. Henningsson, "How TradeLens delivers business value with blockchain technology," MIS Quarterly Executive, 2019.

[9] J. Mattke, A. Hund, C. Maier, and T. Weitzel, "How an enterprise blockchain application in the U.S. Pharmaceuticals supply chain is saving lives," MIS Quarterly Executive, 2019.

[10] R. K. Yin, Case Study Research: Design and Methods. 2009.

[11] M. Hearn, "Corda: A distributed ledger," Corda Technical White Paper, vol. 2016, 2016.

[12] A. Larson, "Partner networks: Leveraging external ties to improve entrepreneurial performance," Journal of business venturing, vol. 6, no. 3, pp. 173-188, 1991.

[13] R. Lapiedra, S. Smithson, J. Alegre, and R. Chiva, "Role of information systems on the business network formation process: An empirical analysis of the automotive sector," Journal of Enterprise Information Management, vol. 17, pp. 219-228, Jan. 2004.

[14] A. B. Pedersen, M. Risius, and R. Beck, "A ten-step decision path to determine when to use blockchain technologies," MIS Quarterly Executive, vol. 18, no. 2, pp. 99-115, 2019.

[15] B. Putz and G. Pernul, "Trust Factors and Insider Threats in Permissioned Distributed Ledgers," Transactions on Large-Scale Data- and Knowledge-Centered Systems, vol. XLII, pp. 25-50, 2019.

[16] R. Ziolkowski, G. Parangi, G. Miscione, and G. Schwabe, "Examining gentle rivalry: Decision-making in blockchain systems," in 52nd Hawaii International Conference on System Sciences, HICSS 2019, Grand Wailea, Maui, Hawaii, USA, January 8-11, 2019 (T. Bui, ed.), pp. 1-10, ScholarSpace, 2019.

[17] R. van Pelt, S. Jansen, D. Baars, and S. Overbeek, "Defining Blockchain Governance: $\{$ A $\}$ Framework for Analysis and Comparison," Inf. Syst. Manag., vol. 38, no. 1, pp. 21-41, 2021.
[18] R. Beck, C. Müller-Bloch, and J. L. King, "Governance in the blockchain economy: A framework and research agenda," Journal of the Association for Information Systems, 2018.

[19] M. Rauchs, A. Blandin, K. Bear, and S. B. McKeon, "2nd Global Enterprise blockchain benchmarking study," Available at SSRN 3461765, 2019.

[20] M. O. Deventer, F. Berkers, M. Vos, A. Zandee, T. Vreuls, L. van Piggelen, A. Blom, B. Heeringa, S. Akdim, P. van Helvoort, et al., "Techruption Consortium Blockchain: What it takes to run a blockchain together," in Proceedings of 1st ERCIM Blockchain Workshop 2018, Amsterdam, Netherlands 8-9 May 2018, European Society for Socially Embedded Technologies (EUSSET), 2018.

[21] M. del Castillo, "Blockchain 50 2021." https: / / www.forbes.com/sites/ michaeldelcastillo/2021/02/02/ blockchain-50/, Feb. 2021.

[22] E. Ganne and D. Patel, "Blockchain \& DLT in Trade: Where do we stand?," 2020.

[23] I. Jacobson, G. Booch, and J. E. Rumbaugh, The Unified Software Development Process - the Complete Guide to the Unified Process from the Original Designers. Addison-Wesley Object Technology Series, Addison-Wesley, 1999.

[24] R. Y. Wang and D. M. Strong, "Beyond Accuracy: What Data Quality Means to Data Consumers," Journal of Management Information Systems, vol. 12, pp. 5-33, Mar. 1996.

[25] R3, "R3 B3i Case Study,” Aug. 2020.

[26] National Bank of Cambodia, "Project Bakong - Next Generation Payment System," tech. rep., Oct. 2020.

[27] R3, "R3 Contour Case Study,” June 2020.

[28] ConsenSys, "Covantis Case Study: Modernizing Global Supply Chains with ConsenSys Blockchain Solutions." https://consensys.net/blockchainuse-cases/global-trade-and-commerce/ covantis/, Oct. 2021.

[29] M. Lacity and R. Van Hoek, "Requiem for reconciliations: DL Freight, a blockchain-enabled solution by Walmart Canada and DLT Labs," 2021.

[30] IBM, "We.trade Case Study." https:// www.ibm.com/case-studies/wetradeblockchain-fintech-trade-finance, 2018.

[31] F. Hawlitschek, B. Notheisen, and T. Teubner, "A 2020 perspective on "The limits of trust-free systems: A literature review on blockchain technology and trust in the sharing economy"," Electron. Commer. Res. Appl., vol. 40, p. 100935, 2020.

[32] E. Abebe, D. Behl, C. Govindarajan, Y. Hu, D. Karunamoorthy, P. Novotny, V. Pandit, V. Ramakrishna, and C. Vecchiola, "Enabling Enterprise Blockchain Interoperability with Trusted Data Transfer (Industry Track)," in Proceedings of the 20th International Middleware Conference Industrial Track, (New York, NY, USA), pp. 29-35, Association for Computing Machinery, 2019.

[33] D. Roeck, H. Sternberg, and E. Hofmann, "Distributed ledger technology in supply chains: A transaction cost perspective," International Journal of Production Research, vol. 58, no. 7, pp. 2124-2141, 2020. 